\title{
A 3D Method for Fit Assessment of a Sizing System
}

\author{
Jiang Wu, Zhizhong Li, and Jianwei Niu \\ Department of Industrial Engineering, Tsinghua University, \\ Beijing, 100084, P.R. China \\ zzli@tsinghua.edu.cn
}

\begin{abstract}
A three-dimensional (3D) method for the evaluation of a sizing system via objective fit assessment is introduced in this study. Taking the evaluation of a helmet sizing system as an example, geometrical models of the human head surfaces of a target population are generated based on 3D anthropometric measurement at first. Then a helmet model for each size is prepared. For each individual, a helmet model of his corresponding size is virtually worn on his head with proper relative position and orientation. After that, objective fit assessment criteria are calculated. Finally, Statistical analysis on these criteria provides an objective evaluation of the sizing system. This method affords a rapid, low-cost, and quantitative approach to carry out fit assessment on a sizing system when critical fit is concerned.
\end{abstract}

Keywords: fit assessment, sizing system, 3D modeling.

\section{Introduction}

Sizing systems provide size specification for different population groups based on some body dimension data from demographic anthropometric surveys and studies. The goal of sizing is to choose limited size groups to cover large percentage of the population. They could be applied to the garment industry, work place design, helmet design, and other operator related products. A sizing system is created by using various methods from trial-and-error to complicated statistical methods. Sizing approaches have been improved and optimization of sizing systems has been developed all along [1]. Statistical methods are widely used to increase accommodation of the population and reduce the number of sizes in the system. For example, in order to establish a pants sizing system, Hsu and Wang performed factor analysis to extract important sizing variables, and used the decision tree technique to identify and classify significant patterns in soldiers' body shape [2].

The head is one of the weakest parts of human body, thus, helmets are broadly used as a protective instruments both in the battlefield and in people's daily work and life i.e., construction worker safety, motorcycle, bicycles, football, baseball, and wrestling. With the increasing demand of good performance and fit of helmet, more and more researchers pay attention to helmet sizing systems. Sippo and Belyavin (1991) introduced a sizing system based on the data of an anthropometric survey on 2000 royal air force aircrew from 1970 to 1971 [3]. Three head dimensions including length, breadth, and pupil-vertex height were used to evaluate the fit of this system. 
Robinette and Whitestone (1992) used dimensions of head length and head width to divide people into several groups [4]. Bradtmiller and Beecher (1993) introduced their new sizing method based on clustering of vectors from the center to the surface of human heads [5]. Chen et al. (2002) proposed a new sizing system using head threedimensional (3D) anthropometric data collected by Computerized Tomography (CT) scanning [6].

Studies indicated that poor fit was the most frequent reason for returning in apparel industry [7]. Bad shoe fit was proved to be the primary reason for sweaty feet, chafing, bunion, and feet discomfort [8]. There are several strategies to improve the fit of products: population grouping, adjustable products, traditional customization, and mass customization [9]. An effective and widely used approach to design well-fit products is to analyze human body forms so as to classify them into several groups.

As mentioned above, sizing is an important way to increase of products fit, and fit is a necessary aspect in a sizing system evaluation. In this study, a method to evaluate a sizing system via objective fit assessment in 3D virtual environment is introduced. This method can give an accurate quantitative assessment with low cost and short development cycle dramatically.

\section{Fit Assessment}

In the product design process, fit testing is expensive but necessary. It has been used to ensure that a certain proportion of a population can wear the clothing or equipment of a given size. Some related guidelines were established by McConvile et al. in 1979 [10]. With helmet system becoming more complex, fit problems which may be ignored before could be threats to performance and individual safety now [11]. Consequently, fit assessment is an important consideration in the evaluation of a sizing system. Many approaches of fit assessment were proposed and implemented. They can be classified into two categories: subjective assessment and objective assessment.

\subsection{Subjective Fit Assessment}

Subjective assessment was more widely used than objective assessment to evaluate the fit of a sizing system. In a typical subjective fit testing, selected subjects are first required to wear prototypes of all available sizes. Experts collect assessment data via questionnaire survey. Two kinds of data are required during a fit testing: anthropometry and fit measuring quality. Statistic methods are used to complete the fit evaluation. Some studies with subjective fit assessment in head sizing systems were carried out. Below are some examples:

In early 1985, the U.S. Army Aeromedical Research Laboratory (1987) initiated an Integrated Helmet and Display Sighting System helmet fitting program. This program aimed to assist the Army to establish fitting requirements and procedures for Advanced Attack Helicopter Program. Questionnaires were used to execute fit assessment in several dimensions including thermal comfort, stability, noise attenuation, ear cups comfort, and chinstrap comfort [12].

The U. S. Army Aeromedical Research Laboratory surveyed the accommodation situation of female hairstyle and flight helmet in 1999 [13]. 
A 24-months experiment assessed current martial helmet in the U. S. Army. Questionnaire survey was distributed to 1,123 soldiers after helmet wearing to evaluate the fit situation [14].

\subsection{Objective Fit Assessment}

In a subjective fit assessment, accurate quantitative criteria for evaluation are difficult to propose due to the complexity and variations of head shapes. Furthermore, the helmets will be continuously worn by subjects till the assessment is finished. If several hours are required for each wearing, the cost, time and patience of subjects in the whole testing may be a big problem. Thus, objective fit assessment becomes interesting to both researchers and practitioners. Two methods of objective fit assessment are broadly used in garment industry. The first one requires comparison between garments and human body linearly. In the other one, the amount of pressure placed by a garment on a specific body location is measured [15]. Using technologies of graphical presentation and analysis of the spatial relationship between helmet and human head, helmet fit can be evaluated in a more accurate degree [16]. This is an important reason why more and more objective fit assessments have been implemented.

The Euclidean distance is the most accepted criterion for objective fit evaluation of a sizing system [17] [18]. In the study of Meunier et al. (2000), fit was assessed by the distance between the insider of the helmet and the surface of the human head [16]. Two approaches were conducted and compared. One was to measure distance using depth probes through holes drilled in the helmet. The other was to calculate the distance via surface data of human head and helmet captured by 3D laser scanning. It was difficult to determine which method yielded the truest results. A computer-aided helmet fit testing reported by Corner et al. (1997) used distances between the interface of a helmet and the surface of a head to draw a fit color map [19].

Many other criteria have been used besides Euclidean distance. In the study executed by Chang, et al. (2001), stability was proved to be enhanced if the helmet fitted the head better and consequently the rotation moments were reduced. Pressure exerted by the helmet on the head can be measured by pressure sensors between helmet and head for objective fit assessment.

\section{3D Fit Assessment Method}

As mentioned above, objective methods give an opportunity to obtain more accurate data for quantitative assessment at a lower cost of time and money than subjective methods. With the development of 3D scanning and Computer-Aided Design (CAD) technologies, objective fit assessment can be done in a virtual environment. Cost can be reduced, and the assessment cycle can be shortened dramatically. Furthermore, the geometric spatial relationship between helmet and head is visible.

A block-distance based sizing method proposed by Niu (in press) considering 3D control points of head surfaces is supposed to be more effective for fitting design than the traditional head sizing methods [20]. Carrying fit assessment on this sizing system will be taken as an example to introduce the proposed 3D fit assessment method. Taking helmet sizing as an example, this method involves 3D geometric modeling of 
subject heads from a 3D anthropometric database and helmets of sizes, head-helmet mating, and fit calculation.

\subsection{D Human Head Modeling}

Since no physical subjects participated in this virtual assessment, 3D human head models were critically important for the accuracy of results [10]. This emphasizes the quality $3 \mathrm{D}$ anthropometric measurement. Then various geometric modeling methods can be used for the modeling of the head. A convenient way for the modeling is to use free form modeling functions of a CAD software, such as Unigraphics which was used in our study. In our case study, the 3D human head data of 510 young male Chinese soldiers was collected by a Chinese military institute in 2002 [6]. Noise data was removed manually by visual check under the CAD environment before the modeling. The head postures of some subjects were not properly settled for scanning, so their postures were standardized before mating with helmet models.

According to the China National Military Standard GJB 5477-2006 [21], the size number was set as three. The 3D head samples were classified by a computer program for the block-distance based sizing [20] into three groups. For each group, a representative $3 \mathrm{D}$ head model was generated by calculating mean values of all samples in this group. These representative head models were used as helmet design reference.

\subsection{D Helmet Modeling}

With the development of CAD technology, it is no longer difficult to set up a helmet model in a virtual environment any more. If the purpose of fit assessment is to evaluate current products, then reverse engineering technology can be adopted, basically involving 3D scanning and surface modeling from a point cloud. While if the purpose is to evaluate a sizing system that corresponding products are to be designed, CAD modeling functions have to be called for creating new models of product sizes. For helmet products, , a rapid computer-aided helmet shell design method based on 3D head anthropometric data under Unigraphics system was proposed and a corresponding toolkit was developed by Liu, et al. (2007) [22]. This toolkit was adopted in our case study to establish the three helmet models for the three sizes.

\subsection{Head-Helmet Mating and Fit Assessment}

The spatial relationship between a head and a helmet should be set up first. According to the right wearing requirement and experiences of experts, every virtual head wore its corresponding helmet virtually with proper relative position and orientation (see Fig.1). Because the head postures have been standardized, the mating mainly involves translational movement for equal gapping between a helmet and a head. Visual observation at the sagittal and lateral sections can help the mating.

Four groups of criteria will be adopted in the fit assessment and comparison between the 3D clustering method and traditional helmet sizing system: head-helmet distance/gap, mass/weight, center of mass, and inertial moments of helmet. The mean value, extreme value, and variance of each criterion are to be calculated. Statistical testing, e.g. Analysis of Variance (ANOVA), on these criteria will be conducted for the comparison. 


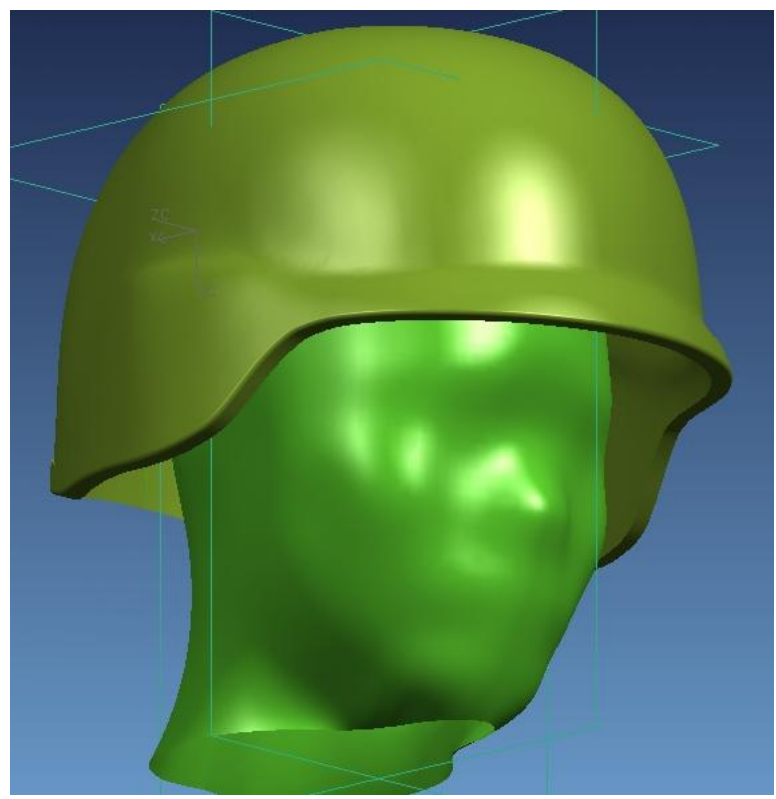

Fig. 1. Spatial relationship between head and helmet

Two kinds of distances will be calculated: the minimum distance and fixed points distances between the external head model surface and the inner helmet surface. Colors will be applied to help visualize the acceptability of the distances.

In a physical experiment, the number of fixed points on the helmet is always limited due to the volume of holes and measure tools. In the experiment by Meunier (2000), only 13 points were drilled in a helmet [16]. On the contrary, more fixed points can be tested on virtual helmet models. Taking the advantages of strong calculation ability of a computer, selecting points evenly distributed on a helmet and calculating the gap at these positions are easy with the help of CAD software.

In this study, 100 points will be tested. By a dedicated computer program, the distances at these 100 fixed points and the minimum distance will be calculated for each head mated with the helmet of the corresponding size virtually. If the distances are between 15 and $25 \mathrm{~mm}$ (GJB 5691-2006) [23], the relevant lines pointing from the helmet surface point to the head surface in a normal direction will be colored green which means acceptable fit. Otherwise, the lines will be colored red.

By this method, the spatial relationship and fit condition between the head and the helmet can be easily seen on the computer. The number of red lines can also be used for fit assessment.

The lighter a helmet is, the more comfortable a wearer will feel, and the less fatigue his/her muscles will have. The function to calculate the mass of a solid model is often available in a 3D CAD software.

Once the mass centers of human head and helmet are inconsistent, the stability of a helmet will be reduced if the vertical distance between the mass centers of the helmet and the head is long. The mass center of a helmet is often higher than a head, so the 
shorter the distance is, the more comfortable the wearing should be. Estimation of mass center of a model is also often available in a 3D CAD software.

\section{Conclusion}

Objective fit assessment can support quantitative calculation and comparison to evaluate a sizing system. With the help of 3D scanning and CAD technologies, objective fit assessment can be done in a virtual environment. Compared with physical assessment and subjective assessment, this method can afford accurate results with dramatically reduced cost. The $3 \mathrm{D}$ graphics visualize how an individual wears the helmet, and give a hand in comprehensive fit assessment. This method can also support fit assessment on a large population, provided that the 3D anthropometric database of this population is available.

\section{Acknowledgements}

This study is supported by the National Natural Science Foundation of China (No.70571045).

\section{References}

1. McCulloch, C.E., Paal, B., Ashdown, S.A.: An optimization approach to apparel sizing. Journal of the Operational Research Society 49(5), 492-499 (1998)

2. Hsu, C.H., Wang, M.J.J.: Using decision tree based data mining to establish a sizing system for the manufacture of garments. International Journal of Advanced Manufacturing Technology 26(5-6), 669-674 (2005)

3. Sippo, A.C., Belyavin, A.J.: Determining aircrew helmet size design requirements using statistical analysis of anthropometric data. Aviation, Space, and Environmental Medicine 66(1), 67-74 (1991)

4. Robinette, K.M., Whitestone, J.J.: Methods for characterizing the human head for the design of helmets. AL-TR-1 992-0061. Armstrong Laboratory, Wright-Patterson AFB OH (1992)

5. Bradtmiller, B., Beecher, R.M.: An approach to creating three-dimensional head forms for helmet sizing and design. In: 31st Annual Symposium Proceedings, pp. 244-249. SAFE Association, Yoncalla (1993)

6. Chen, X., Shi, M.W., Zhou, H., Wang, X.T., Zhou, G.T.: The "standard head" for sizing military helmet based on computerized tomography and the head form sizing algorithm (in Chinese). Acta Armamentrarii 23(4), 476-480 (2002)

7. Iowa Cooperative Extension Service: Consumer choices: finding your best fit (1996), http: / /www. extension. iastate. edu/Publications / PM1648.pdf

8. Rossi, W.A.: The futile search for the perfect shoe fit. Journal of Testing and Evaluation 16, 393-403 (1988)

9. Li, Z.Z.: Anthropometric topography. In: Karwowski, W. (ed.) International Encyclopedia of Ergonomics and Human Factors, 2nd edn. Taylor and Fancis, London (2005) 
10. McConvile, J.T., Tebbetts, I., Alexander, M.: Guidelines for Fit Testing and Evaluation of USAF Personal-Protective Clothing and Equipment, AAMRL-TR-79-2, AD AO 65901, Aerospace Medical Research Laboratory, Aerospace Medical Division, Air Force Systems Command, Weight-Patterson Air Force Base, OH 45433 (1979)

11. Robinette, K.M.: Fit Testing as a Helmet Development Tool. In: Proceedings of the Human Factors and Ergonomics Society 37th Annual Meeting, pp. 69-73 (1993)

12. Rash, C.E., Martin, J.S., Gower Jr., D.W., Licina, J.R., Barson, J.V.: Evaluation of the US Army Fitting Program for the Integrated Helmet Unit of the Integrated Helmet and Display Sighting System, AD-A191616. Fort Rucker. The U.S. Army Aeromedical Research Laboratory, Alabama (1987)

13. McEntire, B.J., Murphy, B.A., Mozo, B.T., Crowley, J.S.: Female Hairstyle and Flight Helmet Accommodation: The AMELIA Project. Fort Rucker. The U. S. Army Aeromedical Research Laboratory, Alabama (1999)

14. Ivins, B.J., Schwab, K.A., Crowley, J.S., McEntire, B.J., Trumble, C.C., Brown, C.F.H., Warden, D.L.: How satisfied are soldiers with their ballistic helmets? A comparison of soldiers' opinions about the advanced combat helmet and the personal armor system for ground troops helmet. Military Medicine 172(6), 586-591 (2007)

15. Ashdown, S.P., Loker, S.: Use of Body Scan Data to Design Sizing Systems Based on Target Markets. National Textile Center Project: S01-CR01 (2002), http: / /www.ntcresearch.org/pdf-rpts/Bref0604/ S01-CR01-04e.pdf

16. Meunier, P., Tack, D., Ricci, A., Bossi, L., Angel, H.: Helmet accommodation analysis using 3D laser scanning. Applied Ergonomics 31, 361-369 (2000)

17. Meunier, P., Yin, S.: Performance of a 2D image-based anthropometric measurement and clothing sizing system. Applied Ergonomics 31, 445-451 (2000)

18. Luximon, A., Goonetilleke, R.S., Zhang, M.: 3D foot shape generation from 2D information. Ergonomics 48(6), 625-641 (2005)

19. Corner, B., Beecher, R., Paquette, S.: Computer-aided fit testing: an approach for examining the user/equipment interface. SPIE 3023, 37-47 (1997)

20. Niu, J.W., Li, Z.Z., Salvendy, G.: Multi-resolution shape description and clustering of three-dimensional head data. Ergonomics (accepted)

21. GJB 5477-2006. 3D Head-face Dimensions of Male Soldiers (in Chinese)

22. Liu, H., Li, Z.Z., Zheng, L.: Rapid preliminary helmet shell design based on threedimensional anthropometric head data. Journal of Engineering Design 19(1), 45-54 (2007)

23. GJB 5691-2006. The Sizes for Military Helmets (in Chinese) 